\title{
Evaluation of genetic diversity and conservation priorities for Egyptian chickens
}

\author{
Sherif Ramadan ${ }^{1,2}$, Boniface B. Kayang ${ }^{3}$, Eiji Inoue ${ }^{4}$, Keijiro Nirasawa ${ }^{5}$, Hiroshi Hayakawa ${ }^{6}$, \\ Shin'ichi Ito ${ }^{7}$, Miho Inoue-Murayama ${ }^{1^{\star}}$ \\ ${ }^{1}$ Wildlife Research Center of Kyoto University, Kyoto, Japan; ${ }^{*}$ Corresponding Author: mmurayama@,wrc.kyoto-u.ac.jp \\ ${ }^{2}$ Faculty of Veterinary Medicine, Benha University, Moshtohor, Egypt \\ ${ }^{3}$ Department of Animal Science, College of Agriculture and Consumer Sciences, University of Ghana, Legon, Ghana \\ ${ }^{4}$ Graduate School of Science, Kyoto University, Kyoto, Japan \\ ${ }^{5}$ Animal Breeding and Reproduction Research Team, National Institute of Livestock and Grassland Science, Tsukuba, Japan \\ ${ }^{6}$ Poultry Research Department, Gifu Prefectural Livestock Research Institute, Seki, Japan \\ ${ }^{7}$ Faculty of Applied Biological Sciences, Gifu University, Gifu, Japan
}

Received 23 April 2012; revised 27 May 2012; accepted 7 June 2012

\begin{abstract}
In this study, 21 microsatellite markers were used to genotype 196 Egyptian local chickens obtained from Fayoumi $(n=35)$, Dandarawy $(n=$ $30)$, Baladi $(n=29)$, Sinai $(n=30)$, El-Salam $(n=$ $36)$, and Golden Montazah $(n=36)$ strains. The results were compared to two pure commercial chicken populations reared in Japan-White Leghorn $(n=42)$ and Rhode Island Red $(n=43)$. A total of 162 alleles were observed, with an average of 7.7 alleles per locus. The average expected heterozygosity for the Egyptian chickens was 0.595 . The closest pairwise Nei's genetic distance was recorded between Sinai and Golden Montazah (0.038) and the smallest pairwise $F_{S T}$ value (0.006) was observed between Baladi and Sinai. The most probable structure clustering of the eight studied populations was at $K=6$. Baladi, Sinai and Golden Montazah strains were clustered together forming admixed mosaic cluster. Dandarawy ranked firstly and contributed the most to aggregate genetic diversity based on two prioritization methods. The information resulting from this study may be used as an initial guide to design further investigations for development of sustainable genetic improvement and conservation programs for the Egyptian chicken genetic resources.
\end{abstract}

Keywords: Conservation; Egyptian Chickens; Genetic Diversity; Microsatellite; Population Structure

\section{INTRODUCTION}

Conservation of genetic diversity is one of the main current issues in the conservation biology literature [1] Conservation is not only about endangered breeds but also about those that are not being utilized efficiently [2]. More than 7500 different breeds of livestock are recognized globally [3]. Conservation of all livestock breeds is considered to be financially infeasible [4], so that priorities need to be set on which population/breed is to be conserved. Both genetic diversity and non-genetic criteria are important for prioritizing breeds for conservation. The non-genetic criteria include threat status and breed merit. The threat status includes risk of extinction and efficiency of the breed utilization, and breed merit includes economic or productive, ecological and sociocultural values of the breeds [5]. As a result of many years of domestication and breeding, a wide variety of chicken breeds exist today. However, an increasing number of local breeds are under threat of extinction and valuable genotypes and traits are at risk of being lost [6]. The genetic erosion of these local breeds may lead to the loss of valuable genetic variability in specific characteristics that are momentarily unimportant in commercial breeding strategies [7].

Egyptian local chickens are subdivided into three groups according to their external morphology [8]. The first group includes pure native breeds, as Fayoumi and Dandarawy. The second group includes mongrel fowl, such as the Baladi and Sinai strains, which originated from hybridization among exotic and Egyptian autochthonous chickens continued along with different times of old trade dispersal and colonization to Egypt. The third group includes improved local strains which originated from crossing between local and standardized exotic chicken strains accompanied by selection for fast growth, such as El-Salam strain [9] and for high egg production, such as Golden Montazah strain [10]. With regard to the commercial sector in Egypt, commercial broilers have 
contributed $63 \%$ of the total poultry production in 2005 . This could reflect the substantial growing of commercial chicken industries in Egypt at the expense of native chicken resources, improvement and maintenance [8]. Egypt possesses versatile varieties of chickens including local types highly adapted to harsh conditions and thought to constitute genetic reservoirs. For instance, the Fayoumi breed has been demonstrated by several studies to possess increased resistance to coccidiosis [11] and Marek's disease [12], and can thus be seen as a unique breed from the viewpoint of disease resistance [12]. Similarly, there is evidence for superiority in heat tolerance, of Sinai strain over White Leghorn and broiler chicks [13].

In Egypt, microsatellites marker analyses were involved in some recent studies to assess genetic diversity within and between local chicken strains $[14,15]$. In this study, we evaluated the genetic diversity and the breed contribution to aggregate genetic diversity as an important criterion for its conservation by utilizing three different prioritization methods in order to set the priorities for conservation of Egyptian chickens based on microsatellite genetic markers.

\section{MATERIALS AND METHODS}

\subsection{Sample Collection and DNA Extraction}

Feather samples were obtained from a total of 196 birds from six Egyptian local chicken strains: Fayoumi $(n=35)$, Dandarawy $(n=30)$, Baladi $(n=29)$, Sinai $(n=$ $30)$, El-Salam $(n=36)$, and Golden Montazah $(n=36)$. For comparative purpose, samples were also obtained from two exotic pure chicken breeds: White Leghorn (WL, $n=42$ ) and Rhode Island Red (RIR, $n=43$ ). The Egyptian samples were collected from Al-Azzab poultry farms belonging to the Poultry Integrated Project at the Fayoum governorate, Egypt. Flock sizes for each breed were about 5000 birds, with sex ratios of one rooster per ten hens. Samples of White Leghorn were collected from the National Institute of Livestock and Grassland Science, Tsukuba, Japan and those of Rhode Island Red from Gifu Prefectural Livestock Research Institute, Gifu, Japan. DNAs were extracted from feather samples using the QIAGEN DNeasy Tissue Kit (QIAGEN, Valencia, CA, USA).

\subsection{Microsatellite Genotyping}

Molecular genotyping of the samples was carried out with a set of 21 autosomal (CA)n di-nucleotide microsatellite markers that are as uniformly distributed as possible throughout the chicken genome. These markers are from the revised set of microsatellites originally recommended by the FAO MoDAD project

(http://www.fao.org/AG/AGAInfo/programmes/en/geneti
cs/documents/ITWG3 Inf3.pdf) for diversity studies in chicken. These markers were used in multiplex PCR reactions employing the QIAGEN Multiplex PCR Kit (QIAGEN, Valencia, CA, USA). PCR was carried out in $10 \mu \mathrm{l}$ reactions containing $20 \mathrm{ng}$ of DNA template, $0.2 \mu \mathrm{M}$ of each primer, of which the forward ones were fluorescently labelled (6-FAM, NED, and HEX) and 2x QIAGEN Multiplex PCR Master Mix. After an initial incubation of $95^{\circ} \mathrm{C}$ for $15 \mathrm{~min}, \mathrm{PCR}$ amplification was performed for 35 cycles consisting of $94^{\circ} \mathrm{C}$ for $30 \mathrm{sec}, 60^{\circ} \mathrm{C}-63^{\circ} \mathrm{C}$ annealing for $90 \mathrm{sec}, 72^{\circ} \mathrm{C}$ for $60 \mathrm{sec}$, followed by a final extension of $60^{\circ} \mathrm{C}$ for $30 \mathrm{~min}$. Subsequently, the PCR products were electrophoresed on an ABI $3130 \times 1$ DNA Sequencer (Applied Biosystems) and the size of fragments was estimated based on 400 HD Rox size marker using the GENEMAPPER software (Applied Biosystems).

\subsection{Data Analysis}

Genetic diversity was assessed by calculating the observed and effective number of alleles $\left(N_{A}\right.$ and $\left.N_{e}\right)$, mean number of alleles $\left(M N_{A}\right)$, observed heterozygosity $\left(H_{O}\right)$ and expected heterozygosity $\left(H_{E}\right)$ by using GENALEX version 6.0 [16]. Polymorphism information content (PIC) was calculated by using Molkin version 2.0 [17]. $F$-statistics [fixation coefficient of an individual within a subpopulation $\left(F_{I S}\right)$, fixation coefficient of an individual within the total population $\left(F_{I T}\right)$, and fixation coefficient of a subpopulation within the total population $\left.\left(F_{S T}\right)\right]$ per locus, in addition to pairwise $F_{S T}$ [18] across the eight studied populations were calculated using GENEPOP version 3.4 [19]. Genetic distances among the eight populations were evaluated by Nei's genetic distance [20]. A phylogenetic tree was constructed based on the Nei's genetic distance $\left(D_{A}\right)$ by using the neighbor-joining (NJ) method [21]. The robustness of tree topologies was evaluated with a bootstrap test of 1000 resampling across loci. These processes were conducted using POPULATIONS version 1.2.30 software (http://bioinformatics-org/ tryphon/populations/).

We investigated the genetic structure of the sampled populations using a Bayesian clustering procedure implemented in STRUCTURE with the admixture method. We analyzed the clustering of the eight studied populations by using independent allele frequencies model [22]. We did 50 runs for each different value of $K(2 \leq K \leq 8)$ with 60,000 iterations following a burn-in period of 100,000 . Pairwise comparisons of the 50 solutions of each $K$ value were run along with 50 permutations using CLUMPP software [23]. The software calculated the highest pairwise similarity index $(H)$. CLUMPP software also outputs a mean of the permuted matrices across replicates after aligning the cluster membership coefficients of these replicate. Finally, the clustering pattern with the 
highest $H$ value was graphically displayed for the selected $K$ value using DISTRUCT software [24]. The most probable clustering numbers (best $\Delta K$ value) was assessed according to the equation [25]:

$$
\Delta K=m\left(\left|L^{\prime \prime}(K)\right|\right) / s[L(K)]
$$

STRUCTURE software identified the migrants and admixed individuals. If the membership coefficient of an individual was more than 0.80 , it was assigned to the cluster completely. If the value was lower than 0.80 , it indicated that the individual was admixed and assigned it to two or more population clusters. The membership coefficient could also identify the migrants who had infiltrated into other chicken population clusters [26].

Different prioritization methods were utilized through measuring the breed contribution to aggregate genetic diversity as in the following:

1) According to Ollivier and Foulley [27], the contribution to between-breed diversity $(C B)$ was computed by estimation of Weitzman values [28] based on the Nei's genetic distance [20] with WEITZPRO [29]. Within breed contributions to diversity $(\mathrm{CW})$ were calculated using the average values of within-breed expected heterozygosity as in the formula: $H_{k}=1-H(S / k) / H(S)$, where $H_{k}$ is the contribution to within-breed diversity $(C W)$ of breed $k, H(S)$ is the average internal heterozygosity of the whole set $S$ and $H(S / k)$ the average internal heterozygosity of the set excluding breed $k$. The aggregate diversity $(D 1)$ was obtained after weighting $C B$ by $F_{S T}$ and $C W$ by $1-F_{S T}$ according to the following equation: $D 1=F_{S T} \mathrm{CB}+\left(1-F_{S T}\right) \mathrm{CW}$. Positive contributions to diversity from a given population using the Ollivier and Foulley [27] method means that the remaining dataset decreases the overall diversity; consequently, the assessed population would be preferred for conservation.

2) According to Petit et al. [30], the rarefacted number of alleles per locus $(k)$ was used to assess the contribution of the $i^{\text {th }}$ population to the total allelic richness as $C_{T}$ $=C_{S}+C_{D}$ where $C_{S}$ is the contribution to the total allelic richness due to the allelic richness of the $i^{\text {th }}$ population and $C_{D}$ is the contribution due to its divergence. Positive contributions to diversity from $i^{\text {th }}$ population using the method of Petit et al. [30] mean that the remaining set has a lower number of alleles than the original set; consequently, the $i^{\text {th }}$ population would be preferred for conservation. This procedure was computed using Molkin version 2.0 [17].

3) According to Caballero and Toro [31], the partitions of the total gene diversity was calculated as in the following equation: $(1-f)=(1-t)+D$, where $f$ is the average global coancestry; $t$ is the average coancestry between populations; $(1-f)=G D_{T}$ representing the total gene diversity; $(1-t)=G D_{w}$, representing the within population component; and $D$ is the Nei genetic distance between populations, representing the between population component. Positive contributions to diversity from a given population using the method of Caballero and Toro [31] mean that the remaining dataset increases the overall diversity; consequently, the assessed population would not be preferred for conservation. This procedure was computed using Molkin version 2.0 [17].

\section{RESULTS AND DISCUSSION}

\subsection{Marker Polymorphisms and Population Diversity}

A total of 162 alleles were observed across all the eight populations, out of which 144 alleles (144/162, $88.9 \%$ ), including 18 unique ones (18/144, 12.5\%), were observed in the six Egyptian populations (Tables 1 and 2). In this study, across the six Egyptian populations the estimated means of $N_{A}(6.9), N_{e}(3.0)$ and $H_{E}(0.595)$ are relatively lower than those of Eltanany et al. [15] who reported values of 7.34, 3.00 and 0.653 , respectively, across ten Egyptian chicken strains using 29 microsatellites loci. The $F_{S T}$ value across the 21 studied loci showed a relatively high mean (0.082) indicating that there is genetic differentiation among the six Egyptian local strains. The estimated $F_{S T}$ value was lower than that measured between pure-bred commercial chicken lines in a study in Zimbabwe which showed 0.357 of total genetic variation owing to line differences [32]. However, it was slightly higher than the 0.068 previously reported across ten Egyptian chicken strains [15]. In this study, $F_{S T}$ recorded a high value $(0.222)$ after adding the two exotic pure populations (WL and RIR) indicating that, there is high genetic differentiation between these two exotic pure breeds and Egyptian chicken populations (Table 1). The relatively low but positive $F_{I S}$ average $(0.051)$, in addition to the eleven loci showing a deficit of heterozygote might indicate non-random mating and also these loci might be under morphological or productive traits of selective interest. Moreover, $F_{I S}$ is used to obtain a deeper insight to appraise the degree of inbreeding and endangerment potentiality and is considered as an important tool to judge the conservation priority [33]. Accordingly, when $F_{I S}$ is less than 0.05 , the breeds are not in danger; between $0.05-0.15$, they are potentially endangered; between $0.15-0.25$, they are minimally endangered; between $0.25-0.40$, they are endangered; and more than 0.40 , they are critically endangered. In this study, Fayoumi, Dandarawy, El-Salam and RIR populations showed high levels of inbreeding $(0.110,0.053,0.095$ and 0.083 , respectively), posing their potential endangerment [33]. 
Table 1. Observed $\left(N_{A}\right)$ and effective $\left(N_{e}\right)$ number of alleles, polymorphism information content $(P I C)$, observed $\left(H_{O}\right)$ and expected $\left(H_{E}\right)$ heterozygosities, and $F$-statistics $\left(F_{I S}, F_{S T}\right.$, and $\left.F_{I T}\right)$ across the six Egyptian strains.

\begin{tabular}{|c|c|c|c|c|c|c|c|c|}
\hline Locus & $N_{A} \pm S D$ & $N_{e} \pm S D$ & $P I C \pm S D$ & $H_{o} \pm S D$ & $H_{E} \pm S D$ & $F_{I S} \pm S E$ & $F_{S T} \pm S E$ & $F_{I T} \pm S E$ \\
\hline$A D L 268$ & 6.0 & 4.0 & 0.767 & 0.686 & 0.751 & 0.093 & 0.070 & 0.156 \\
\hline$A D L 278$ & 4.0 & 2.5 & 0.596 & 0.612 & 0.594 & -0.034 & 0.132 & 0.102 \\
\hline$A D L 112$ & 3.7 & 2.0 & 0.426 & 0.441 & 0.436 & -0.014 & 0.115 & 0.102 \\
\hline$M C W 295$ & 4.8 & 2.0 & 0.467 & 0.337 & 0.457 & 0.251 & 0.087 & 0.316 \\
\hline$M C W 216$ & 3.2 & 2.1 & 0.465 & 0.408 & 0.518 & 0.217 & 0.044 & 0.251 \\
\hline$M C W 014$ & 3.0 & 1.4 & 0.276 & 0.128 & 0.265 & 0.540 & 0.092 & 0.582 \\
\hline$M C W 098$ & 2.0 & 1.3 & 0.192 & 0.224 & 0.216 & -0.025 & 0.035 & 0.011 \\
\hline LEI234 & 10.0 & 5.7 & 0.851 & 0.638 & 0.817 & 0.220 & 0.069 & 0.273 \\
\hline$M C W 111$ & 4.7 & 2.8 & 0.613 & 0.631 & 0.628 & -0.004 & 0.055 & 0.052 \\
\hline$M C W 078$ & 4.0 & 2.0 & 0.511 & 0.521 & 0.491 & -0.053 & 0.201 & 0.159 \\
\hline$M C W 222$ & 3.8 & 1.9 & 0.478 & 0.458 & 0.472 & 0.036 & 0.122 & 0.154 \\
\hline$M C W 183$ & 8.0 & 5.3 & 0.843 & 0.712 & 0.811 & 0.121 & 0.068 & 0.180 \\
\hline LEI094 & 9.8 & 5.2 & 0.837 & 0.778 & 0.814 & 0.051 & 0.060 & 0.108 \\
\hline$M C W 069$ & 5.5 & 3.4 & 0.700 & 0.659 & 0.698 & 0.047 & 0.067 & 0.111 \\
\hline$M C W 034$ & 6.7 & 3.9 & 0.716 & 0.793 & 0.722 & -0.098 & 0.050 & -0.044 \\
\hline$M C W 037$ & 3.0 & 2.6 & 0.555 & 0.622 & 0.613 & -0.015 & 0.038 & 0.024 \\
\hline$M C W 067$ & 3.2 & 2.6 & 0.572 & 0.601 & 0.615 & 0.027 & 0.059 & 0.085 \\
\hline$M C W 206$ & 5.5 & 3.0 & 0.673 & 0.704 & 0.673 & -0.042 & 0.088 & 0.050 \\
\hline$M C W 081$ & 5.7 & 3.5 & 0.699 & 0.684 & 0.676 & -0.010 & 0.092 & 0.083 \\
\hline LEI166 & 3.2 & 2.4 & 0.563 & 0.615 & 0.579 & -0.067 & 0.114 & 0.055 \\
\hline$M C W 330$ & 4.0 & 3.0 & 0.644 & 0.636 & 0.653 & 0.027 & 0.085 & 0.110 \\
\hline Mean & $6.9 \pm 3.6$ & $3.0 \pm 0.6$ & $0.593 \pm 0.175$ & $0.566 \pm 0.092$ & $0.595 \pm 0.078$ & $0.051 \pm 0.032$ & $0.082 \pm 0.008$ & $0.129 \pm 0.029$ \\
\hline Total mean ${ }^{\mathrm{a}}$ & $7.7 \pm 4.2$ & $2.8 \pm 0.5$ & $0.649 \pm 0.137$ & $0.536 \pm 0.078$ & $0.564 \pm 0.069$ & $0.051 \pm 0.018$ & $0.222 \pm 0.023$ & $0.261 \pm 0.026$ \\
\hline
\end{tabular}

a Total mean includes WL and RIR in addition to the six Egyptian breeds.

Table 2. Mean observed $\left(M N_{A}\right)$ and effective $\left(M N_{e}\right)$ number of alleles, unique alleles, observed $\left(H_{O}\right)$ and expected $\left(H_{E}\right)$ heterozygosities, and fixation coefficient of an individual within a subpopulation $\left(F_{I S}\right)$ per breed.

\begin{tabular}{lccccccc}
\hline \multicolumn{1}{c}{ Breed/strain } & $n$ & $M N_{A} \pm \mathrm{SD}$ & $N_{e} \pm \mathrm{SD}$ & Unique alleles & $H_{o} \pm \mathrm{SD}$ & $H_{E} \pm \mathrm{SD}$ & $F_{I S} \pm \mathrm{SE}$ \\
\hline Egyptian strains & 196 & $4.9 \pm 0.5$ & $3.0 \pm 1.4$ & 18 & $0.566 \pm 0.049$ & $0.595 \pm 0.042$ & $0.053 \pm 0.017$ \\
Fayoumi & 35 & 3.7 & 2.3 & 0 & $0.423 \pm 0.215$ & $0.475 \pm 0.220$ & 0.110 \\
Dandarawy & 30 & 4.4 & 2.7 & 4 & $0.560 \pm 0.197$ & $0.591 \pm 0.147$ & 0.053 \\
Baladi & 29 & 5.9 & 3.4 & 9 & $0.622 \pm 0.211$ & $0.645 \pm 0.192$ & 0.036 \\
Sinai & 30 & 5.4 & 3.4 & 2 & $0.648 \pm 0.179$ & $0.660 \pm 0.142$ & 0.020 \\
El-Salam & 36 & 4.8 & 2.9 & 0 & $0.527 \pm 0.243$ & $0.582 \pm 0.211$ & 0.095 \\
Golden Montazah & 36 & 5.4 & 3.2 & 3 & $0.616 \pm 0.224$ & $0.618 \pm 0.202$ & 0.003 \\
WL & 42 & 2.5 & 2.0 & 4 & $0.423 \pm 0.234$ & $0.428 \pm 0.229$ & 0.012 \\
RIR & 43 & 3.6 & 2.2 & 4 & $0.469 \pm 0.160$ & $0.511 \pm 0.137$ & 0.083 \\
Total Mean & 281 & $4.5 \pm 0.5$ & $2.8 \pm 1.2$ & 26 & $0.536 \pm 0.048$ & $0.564 \pm 0.042$ & $0.052 \pm 0.014$ \\
\hline
\end{tabular}

In respect to the within population genetic diversity, the studied eight chicken populations could be categorized into a low diversity class (Fayoumi, WL, and RIR) and a high diversity class which includes the remaining five populations. This is in agreement with breed history and management. These populations (WL and RIR) had undergone selection for high growth rate (RIR) and high egg production (WL). Moreover, the Fayoumi strain recorded the highest value of $F_{I S}(0.110)$ and complete allele fixation (monomorphic) of the $M C W 014$ locus (Table 2). This might be attributed to its narrow genetic base as it is an ancient native chicken bred as a closed popula- 
tion. The two Egyptian mongrel strains (Baladi and Sinai) recorded the highest genetic diversity $\left(M N_{A}=5.9 ; N_{e}=\right.$ 3.4; $H_{O}=0.622$, and $H_{E}=0.645$ for Baladi and $M N_{A}=$ 5.4; $N_{e}=3.4 ; H_{O}=0.648$, and $H_{E}=0.660$ for Sinai) among the eight studied populations and this might be attributed to their wide genetic bases due to hybridization among exotic and Egyptian autochthonous chickens continued along with different times of old trade dispersal and colonization to Egypt [8].

\subsection{Genetic Relationship}

The Nei's genetic distance $\left(D_{A}\right)$ and pairwise $F_{S T}$ statistic were estimated for the eight studied chicken populations across the 21 microsatellite loci (Table 3). The closest pairwise Nei's genetic distance was recorded between the Sinai and Golden Montazah strains (0.038) and this was supported by clustering in the neighbor-joining phylogenetic tree (Figure 1). Similarly, the lowest pairwise $F_{S T}$ value was recorded between the Baladi and Sinai strains (0.006). The close relation between Sinai and Golden Montazah and also between Baladi and Sinai can be attributed to the mongrel nature of Baladi and Sinai strains which originated from hybridization among exotic and Egyptian autochthonous chickens.

\subsection{Population Structure and Individual's Assignment}

The most probable structure clustering of the eight studied chicken populations was at $K=6$ (Figure 2). The pure breeds (WL, RIR, Fayoumi and Dandarawy) in addition to El-Salam were assigned independently into their respective clusters while the remaining three populations (Baladi, Sinai and Golden Montazah) were clustered together forming admixed mosaic cluster. A probable explanation for the separation of El-Salam to form its own cluster is that it might have experienced high inbreeding and low gene flow from other strains under this study. This is in line with the relatively high $F_{I S}(0.095)$ value within the El-Salam strain. The high genetic admixture and migrations between Baladi, Sinai, and Golden Montazah strains could contribute to gather them forming the admixed mosaic cluster.

Table 3. Nei's genetic distance $\left(\mathrm{D}_{A}\right.$ : above diagonal) and pairwise $F_{S T}$ (below diagonal) estimates for the 21 microsatellite loci between the eight studied chicken strains.

\begin{tabular}{lcccccccc}
\hline & Fayoumi & Dandarawy & Baladi & Sinai & El-Salam & $\begin{array}{c}\text { Golden } \\
\text { Montazah }\end{array}$ & WL & RIR \\
\hline Fayoumi & & 0.185 & 0.104 & 0.143 & 0.165 & 0.155 & 0.506 & 0.501 \\
Dandarawy & 0.170 & & 0.115 & 0.134 & 0.170 & 0.155 & 0.486 & 0.420 \\
Baladi & 0.079 & 0.062 & & 0.040 & 0.081 & 0.059 & 0.469 & 0.419 \\
Sinai & 0.116 & 0.074 & 0.006 & & 0.770 & 0.038 & 0.468 & 0.403 \\
El-salam & 0.137 & 0.118 & 0.043 & 0.051 & & 0.070 & 0.489 & 0.422 \\
Golden Montazah & 0.143 & 0.111 & 0.033 & 0.020 & 0.045 & & 0.494 & 0.401 \\
WL & 0.399 & 0.354 & 0.318 & 0.311 & 0.351 & 0.352 & & 0.326 \\
RIR & 0.392 & 0.316 & 0.285 & 0.268 & 0.299 & 0.279 & 0.302 & \\
\hline
\end{tabular}

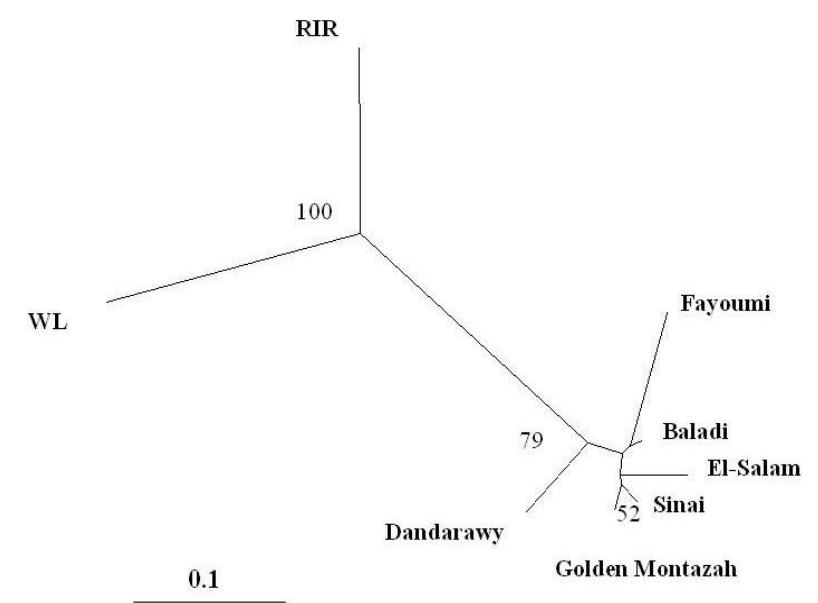

Figure 1. Neighbor-joining tree of the six Egyptian and the two exotic pure chicken populations based on the 21 microsatellite loci. The consensus tree was generated with 1000 bootstraps over loci and bootstrap values lower than 50 are not shown in the diagram. 
The distribution of 35 admixed and five migrant individuals in the inferred six clusters according to their membership coefficients were evaluated (Table 4). All the studied populations recorded more than 0.80 membership coefficients in their inferred clusters except Baladi strain (0.69). Despite that Sinai and Golden Montazah strains had more than 0.80 membership coefficient, they were clustered together in the same cluster with Baladi strain. This might be explained by the genetically similar nature of these strains as confirmed by their pairwise genetic distances (Table 3) and the mosaic admixture in the STRUCTURE dendrogram (Figure 2). Thus, based on the preceding results, when the Baladi, Sinai, and Golden Montazah chicken strains are to be used for future research work it would be necessary to consider, in addition to random selection, the admixed individuals and migrants.

\subsection{Conservation Prioritization of the Studied Strains}

In the current study different prioritization methods were utilized to measure the breed contribution to aggregate genetic diversity as an important criterion for its conservation (Table 5). All such methods revealed that Fayoumi strain contributed negatively to aggregate genetic diversity $(C W=-4.20, D 1=-1.15, D 2=-1.89$ and $G D=1.72$ ). Therefore, Fayoumi according to such a determined criterion may be ranked last for conservation.
On the contrary, Dandarawy contributed the most $(C B=$ $34.92, D 2=2.49, G D=-1.40)$ to aggregate genetic diversity according to $[27,30,31]$. The two Egyptian mongrel strains ranked second $(D 1=2.90, G D=-1.23$ for Sinai and $D 1=1.73, D 2=2.27$ for Baladi), while Egyptian synthetic strains (Golden Montazah then El-Salam) came in the third position according to their contribution to aggregate genetic diversity. The preceding prioritization of the breeds for conservation is based only on molecular genetic marker information, but when we combine other non-genetic criteria the ranking may become different. Thus, according to the preceding prioritization methods, Fayoumi ranked the last, but after considering its high level of inbreeding and breed merit in term of disease resistance ability (Marek's disease and coccidiosis), it may get advanced ranking. Similarly, Sinai strain ranked second, but after considering its breed merit (superiority in heat tolerance) it may get a different ranking.

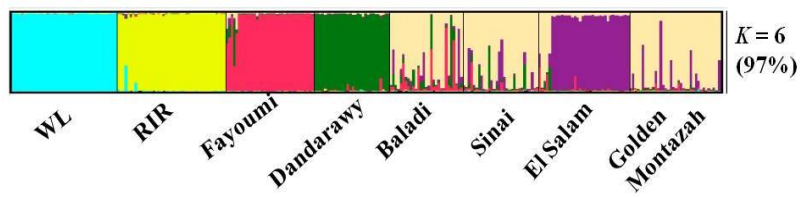

Figure 2. Structure clustering of the six Egyptian and two exotic pure chicken populations obtained for $K=6$. The percentage inside the parenthesis is the average pairwise similarity index $(H)$ of the individuals Q matrix, while $K$ is the cluster number.

Table 4. Number of admixed and migrant individuals in the inferred clusters.

\begin{tabular}{llccc}
\hline Cluster & Strain & Membership coefficient & Admixed individuals & Migrant individuals to another cluster \\
\hline Cluster I & WL & 0.99 & 0 & - \\
Cluster II & RIR & 0.97 & 0 & - \\
Cluster III & Fayoumi & 0.94 & 4 & - \\
Cluster IV & Dandarawy & 0.96 & 1 & - \\
Cluster V & El-Salam & 0.82 & 4 & 2 (Cluster VI) \\
& Baladi & 0.69 & 10 & 2 (Cluster III and Cluster IV) \\
Cluster VI & Sinai & 0.81 & 9 & - \\
& Golden Montazah & 0.86 & 7 & 1 (Cluster V) \\
\hline
\end{tabular}

Table 5. Contribution of each strain to aggregate genetic diversity.

\begin{tabular}{lccccc}
\hline Strain & $C W^{\mathrm{a}}$ & $C B^{\mathrm{b}}$ & $D 1^{\mathrm{c}}$ & $D 2^{\mathrm{d}}$ & $G D^{\mathrm{e}}$ \\
\hline Fayoumi & -4.202 & 33.970 & -1.148 & -1.892 & 1.716 \\
Dandarawy & -0.168 & 34.920 & 2.639 & 2.490 & -1.404 \\
Baladi & 0.840 & 11.950 & 1.729 & 2.273 & -0.414 \\
Sinai & 2.521 & 7.240 & 2.899 & 0.989 & -1.231 \\
El-Salam & -0.504 & 17.430 & 0.931 & 0.196 & 0.194 \\
Golden Montazah & 0.840 & 10.930 & 1.648 & 0.791 & -0.823 \\
\hline
\end{tabular}

${ }^{\mathrm{a}} \mathrm{CW}=$ contribution to within-population genetic diversity; ${ }^{\mathrm{b}} \mathrm{CB}=$ contribution to between-population genetic diversity (Weitzman 1993 ); ${ }^{\mathrm{c}} \mathrm{D} 1=\mathrm{contribution}$ to aggregate genetic diversity (Ollivier and Foulley 2005); ${ }^{\mathrm{d}} \mathrm{D} 2$ = global diversity contribution (Petit et al. 1998); ${ }^{\mathrm{e}} \mathrm{GD}=$ global diversity contribution (Caballero and Toro 2002). 
In conclusion, the results from this study confirm the applicability and efficiency of this microsatellite panel for assessing genetic variation and setting the conservation priorities for Egyptian local chickens. Consideration of breed merits and threat status, in addition to genetic diversity, enabled us to balance the trade-offs between conserving diversity as insurance against future uncertainties and current sustainable utilization. More detailed information about non-genetic aspect (threat status and breed merits) and a conceptual framework for a maximum utility through a weighted summation of measures of neutral diversity, breed merits and threat status of Egyptian chickens merits consideration.

\section{ACKNOWLEDGEMENTS}

We thank Dr. Ahmed Radwan, Director of Fowl Integrated Project, Fayoum Governorate, Egypt, for the great facilities he kindly offered during the feather sampling of Egyptian chickens. We are grateful to Dr. Azusa Hayano, Wildlife Research Center of Kyoto University for her assistance in data analysis. This study was supported financially in part by the Ministry of Education, Culture, Sports, Science and Technology (MEXT) with a Grant-in-aid for Science Research (\#21310150 to MI-M) and the Global Center of Excellence Program "Formation of a Strategic Base for Biodiversity and Evolutionary Research: from Genome to Ecosystem".

\section{REFERENCES}

[1] Frankham, R. (1995) Conservation genetics. Annual Review of Genetics, 29, 305-327. doi:10.1146/-annurev.ge.29.120195.001513

[2] Barker, J.S. (2001) Conservation and management of genetic diversity: A domestic animal perspective. Canadian Journal of Forest Research, 31, 588-595. doi:10.1139/x00-180

[3] FAO (2007) The state of the world's animal genetic resources for food and agriculture. FAO, Rome. http://www.fao.org/docrep/010/a1250e/a1250e00.htm

[4] Bennewitz, J., Eding, H., Ruane, J. and Simianer, H. (2007) Selection of breeds for conservation. In: Oldenbrook, K., Ed., Utilization and Conservation of Farm Animal Genetic Resources, Wageningen Academic Publishers, Wageningen, 131-146.

[5] Ruane, J. (2000) A framework for prioritizing domestic animal breeds for Conservation purposes at the national level: A Norwegian case study. Conservation Biology, 14, 1385-1393. doi:10.1046/j.1523-1739.2000.99276.x

[6] Blackburn, H. (2006) The National Animal Germplasm Program: Challenge and opportunities for poultry genetic resources. Poultry Science, 85, 210-215.

[7] Weigend, S., Vef, E., Wesch, G., Meckenstock, E., Seibold, R. and Ellendorff, F. (1995) Concept for conserving genetic resources in poultry in Germany. Archiv für Geflügelkunde, 59, 327-334.

[8] Hosny, F.A. (2006) The structure and importance of the commercial and village based poultry systems in Egypt. In: Food and Agriculture Organization of the United Nations, Ed., Poultry Sector Country Review, 1-39.

[9] Abd El-Gawad, E.M., Balat, M.M., Abou- El- Ella, N.Y., Ali, M.M. and Omran, Kh.M. (1983) "El-Salam" a new locally developed strain of chickens. Agricultural Research Revolution, 61, 147-157.

[10] Mahmoud, T.H., Sayed, I.F. and Madkour, Y. H. (1974) "The Golden Montazah" a new variety of chickens. $A g$ ricultural Research Revolution Cairo, 52, 97-105.

[11] Pinard-Van Der Laan, M.H., Monvoisin, J.L., Pery, P., Hamet, N. and Thomas, M. (1998) Comparison of outbred lines of chickens for resistance to experimental infection with coccidiosis (Eimeria tenella). Poultry Science, 77, 185-191.

[12] Tixier-Boichard, M., Bordas, A. and Rognon, X. (2009) Characterisation and monitoring of poultry genetic resources. World's Poultry Science Journal, 65, 272-285. doi:10.1017/S0043933909000233

[13] Arad, Z., Marder, J. and Soller, M. (1981) Effect of gradual acclimation to temperatures up to $44^{\circ} \mathrm{C}$ on productive performance of the desert Bedouin fowl, the commercial White Leghorn and the two reciprocal crossbreds. British Poultry Science, 22, 511-520. doi:10.1080/00071688108447918

[14] Roushdy, Kh., Zein El-Dein, A., Fathi, M.M., Ali, U.M. and Assy, H.M. (2008) Microsatellite genetic differentiation analysis of two local chicken breeds compared with foreign Hy-line strain. International Journal of Poultry Science, 7, 1045-1053. doi:10.3923/ijps.2008.1045.1053

[15] Eltanany, M., Philipp, U., Weigend, S. and Distl, O. (2011) Genetic diversity of ten Egyptian chicken strains using 29 microsatellite markers. Animal Genetics, 42, 666-669. doi:10.1111/j.1365-2052.2011.02185.x

[16] Peakall, R. and Smouse, P.E. (2006) GENALEX 6: Genetic analysis in Excel. Population genetic software for teaching and research. Molecular Ecology Notes, 6, 288295. doi:10.1111/j.1471-8286.2005.01155.x

[17] Gutiérrez, J.P., Royo, L.J., Álvarez, I. and Goyache, F. (2005) Molkin v2.0: A computer program for genetic analysis of populations using molecular coancestry information. Journal of Heredity, 96, 718-721. doi:10. 1093/jhered/esi118

[18] Weir, B.S. and Cockerham, C.C. (1984) Estimating F-statistics for the analysis of population structure. Evolution, 38, 1358-1370. doi:10.2307/2408641

[19] Raymond, M. and Rousset, F. (1995) GENEPOP (version 1.2): Population genetics software for exact tests and ecumenicism. Journal of Heredity, 86, 248-249.

[20] Nei, M., Tajima, F. and Tateno, Y. (1983) Accuracy of estimated phylogenetic trees from molecular data. II. Gene frequency data. Journal of Molecular Evolution, 19, 153-170. doi:10.1007/BF02300753

[21] Saitou, N. and Nei, M. (1987) The neighbor-joining method: A new method for reconstructing phylogenetic trees. Molecular Biology and Evolution, 4, 406-425.

[22] Pritchard, J.K., Stephens, M. and Donnelly, P. (2000) 
Inference of population structure using multilocus genotype data. Genetics, 155, 945-959.

[23] Jakobsson, M. and Rosenberg, N.A. (2007) CLUMPP: A cluster matching and permutation program for dealing with label switching and multimodality in analysis of population structure. Bioinformatics, 23, 1801-1806. doi:10.1093/bioinformatics/btm233

[24] Rosenberg, N.A. (2004) DISTRUCT: A program for the graphical display of population structure. Molecular Ecology Notes, 4, 137-138. doi:10.1046/j.1 $471-8286.2003 .00566 . x$

[25] Evanno, G., Regnaut, S. and Goudet, J. (2005) Detecting the number of clusters of individuals using the software STRUCTURE, a simulation study. Molecular Ecology, 14, 2611-2620. doi:10.1111/j.1365-294X. 2005.02553.x

[26] Li, H.F., Han, W., Zhu, Y.F., Shu, J.T., Zhang, X.Y. and Chen, K.W. (2009) Analysis of genetic structure and relationship among nine indigenous Chinese chicken populations by the Structure program. Journal of Genetics, $\mathbf{8 8}$, 197-203. doi:10.1007/s12041-009-0028-8

[27] Ollivier, L. and Foulley, J.L. (2005) Aggregate diversity: new approach combining within and between-breed genetic diversity. Livestock Production Science, 95, 247-254. doi:10.1016/j.livprodsci.2005.01.005

[28] Weitzman, M.L. (1993) What to preserve? An application of diversity theory to Crane conservation. Quarterly Journal of Economics, 108, 157-183.

$$
\text { doi: } 10.2307 / 2118499
$$

[29] Derban, S., Foulley, J.L. and Ollivier, L. (2002) WEITZPRO: A software for analyzing genetic diversity. Institut National de la Recherche Agronomique, Paris. http://www23sgqa.jouy.inra.fr/article.php3?id_article=3

[30] Petit, R.J., El Mousadik, A. and Pons, O. (1998) Identifying populations for conservation on the basis of genetic markers. Conservation Biology, 12, 844-855. doi:10.1046/j.1523-1739.1998.96489.x

[31] Caballero, A. and Toro, M.A. (2002) Analysis of genetic diversity for the management of conserved subdivided populations. Conservation Genetics, 3, 289-299. doi:10.1023/A:1019956205473

[32] Muchadeyi, F.C., Eding, H. Wollny, C.B., Groeneveld, E., Makuza, S.M., Shamseldin, R., Simianer, H. and Weigend, S. (2007) Absence of population substructuring in Zimbabwe chicken ecotypes inferred using microsatellite analysis. Animal Genetics, 38, 332-339. doi:10.1111/j.1365-2052.2007.01606.x

[33] Simon, D.L. and Buchenauer, D. (Eds.) (1993) Genetic diversity of European livestock breeds. Results of Monitoring by the EAAP Working Group on Animal Genetic Resources; EAAP Animal Genetic Data Bank, Institute of Animal Breeding and Genetics, School of Veterinary Medicine, Hannover, EAAP Publication, No. 66, Wageningen Pers, Wageningen, $591 \mathrm{p}$. 